\title{
LORENTZ FORCE MAGNETOMETER WITH QUADRATURE FREQUENCY MODULATION
}

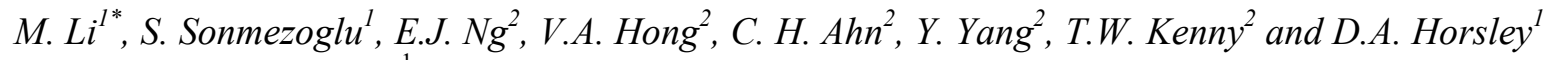 \\ ${ }^{1}$ Univeristy of California, Davis, California, USA \\ ${ }^{2}$ Stanford University, Stanford, California, USA
}

\begin{abstract}
In this paper, a Lorentz force magnetometer demonstrates quadrature frequency modulation (QFM) operation. The Lorentz force magnetometer consists of a conventional 3-port resonator, which is put into oscillation by electrostatic driving and sensing. The bias current flowing through the resonator is proportional to the displacement, and generates Lorentz force in quadrature with the electrostatic force. As a result, the Lorentz force acts as an equivalent spring and the magnetic field can be measured by reading the change in oscillation frequency. The sensor has a sensitivity of $500 \mathrm{~Hz} / \mathrm{T}$ with a short-term noise floor of $500 \mathrm{nT} / \sqrt{\mathrm{Hz}}$. The bandwidth of the sensor is increased to $50 \mathrm{~Hz}$, a factor of 12 greater than that of the same resonator operating in amplitude-modulated (AM) mode.
\end{abstract}

\section{INTRODUCTION}

Many Lorentz force magnetometers have been proposed in recent years [1-3]. The MEMS magnetometer is entirely silicon, sharing the same fabrication process as commercially available accelerometers and gyroscopes. A single-structure 3-axis magnetometer has also been reported [3], giving it great potential to be used as an electronic compass in smart phones. Compared to the commercially available Hall-Effect sensors currently used in smart phones [4], MEMS magnetometers do not require flux-concentrators for 3-axis measurement and are free from magnetic hysteresis.

This work demonstrates a resonant Lorentz force magnetometer with quadrature frequency modulation (QFM) readout. Readout control electronics based on amplitude modulation (AM) and fabrication of the device were reported in [1]. A conventional AM magnetometer modulates low frequency magnetic field to a frequency near the device's resonance frequency. The motion resulting from the Lorentz force is therefore amplified by the mechanical quality factor (Q) of the sensor, and the motion's amplitude is used as a measure of the input magnetic field. Although Brownian-limited noise floor can be achieved, AM magnetometers suffer from small bandwidth and large temperature sensitivity. In an FM magnetometer, input magnetic field results in a change in the oscillation frequency by varying the stiffness $(k)$ of the sensor. Two different methods for FM modulation have been reported in the literature. A straightforward method is to use Lorentz force to generate axial stress [5] which results in a change in $k$ of the oscillator and therefore the resonance frequency of the oscillator changes. The other method is to use quadrature frequency modulation (QFM), where an external force having the same frequency as, but in quadrature with, the self-sustaining force creates a phase shift in the oscillation loop. This phase shift results in a change in the oscillation frequency, since oscillation always occurs at the frequency that satisfies $0^{\circ}$ phase shift around the loop. MEMS QFM gyroscopes (having Coriolis force as the external force) [6] and magnetometers (having Lorentz force as the external force) [7] were demonstrated. Using the same MEMS magnetometer structure, magnetic field can be either amplitude modulated (AM) or frequency modulated (FM) by controlling the phase difference between the Lorentz force and electrostatic force. Relative to earlier AM magnetometers, QFM operation extends the sensor's bandwidth from a few $\mathrm{Hz}$ to $50 \mathrm{~Hz}$, which is independent from the sensor's mechanical bandwidth. QFM operation also significantly increases the dynamic range.

\section{THEORY}

\section{System Dynamics}

The MEMS Lorentz force magnetometer is based on a traditional three-port MEMS resonator operating as an oscillator. The resonance frequency serves as the system reference clock for the bias current generation.

The system dynamics of the Lorentz force magnetometer can be modeled as a second order mass-spring-damper system:

$$
m \ddot{x}+b \dot{x}+k x=F_{E}+F_{L}
$$

where $m$ is the effective mass, $b$ is the damping coefficient, $k$ is the spring constant, $F_{E}$ is the electrostatic driving force and $F_{L}$ is the Lorentz force. In the operation of a QFM magnetometer, $F_{L}$ is always in phase with the displacement $x$ :

$$
F_{L}=B I L=B A_{I} x L
$$

where the bias current gain $A_{I}$ is used here to represent the bias current to displacement ratio, $A_{I}=I / x$, which is controlled by the electronics. By replacing the Lorentz force $F_{L}$ according (2), the equation of motion (1) can be written as

$$
m \ddot{x}+b \dot{x}+\underbrace{\left(k-B A_{I} L\right)}_{k_{e f f}} x=F_{E}
$$

It is clearly seen from (3) that the magnetic field modifies the effective spring constant of the device, $k_{\text {eff }}$. Therefore, when the resonator operates in closed-loop, the magnetic field signal can be observed through a change in oscillation frequency.

To demonstrate the principle of operation, the open-loop force-to-displacement frequency response of the QFM magnetometer is measured. Figure 2 shows the amplitude (top) and phase (bottom) characteristics. In this measurement, the bias current gain is set to $A_{I}=-3200 \mathrm{~A} / \mathrm{m}$. The black trace in Figure 2 shows the response of the magnetometer in the absence of an external magnetic field, whereas the blue and red traces show the
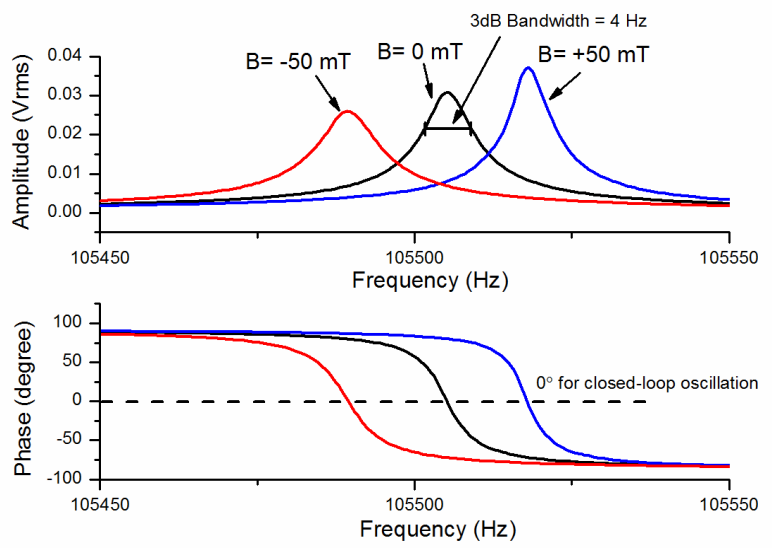

Figure 1: Amplitude (top) and phase (bottom) characteristics of the QFM magnetometer with no external magnetic field applied (black trace) and $\pm 50 \mathrm{~m} T$ magnetic field (blue and red traces) applied. 
response in the presence of $50 \mathrm{mT}$ and $-50 \mathrm{mT}$ magnetic field, respectively. In closed-loop operation, the phase around the oscillation loop is always $0^{\circ}$, as indicated by the black dashed line in the phase characteristic, and changes in magnetic field result in changes in the oscillation frequency. A change in the peak amplitude is also observed when magnetic field is applied, mainly due to the phase error introduced by the detection electronics and bias current generation. If the bias current is not exactly in quadrature with the velocity of the resonator, some part of the Lorentz force will be in phase with the damping term, thus changing the oscillation amplitude and the effective $\mathrm{Q}$ of the resonator. This effect can be suppressed by improving the electronics design.

\section{Sensitivity}

At resonance, the electrostatic force, $F_{E}$, is in phase with the velocity $\dot{x}$, but in quadrature with the Lorentz force, $F_{L}$. Using the effective spring constant, $k_{\text {eff }}=\left(k-B A_{I} L\right)$, the resonance frequency can be represented by

$$
f_{n}=\frac{1}{2 \pi} \sqrt{\frac{k_{e f f}}{m}}
$$

Taking the derivative of (4) with respect to magnetic field $B$, the sensitivity, in units of $\mathrm{Hz} / \mathrm{T}$, can be derived as

$$
S_{B}=\frac{\partial f_{n}}{\partial B}=\frac{-A_{I} L}{4 \pi \sqrt{m\left(k_{e f f}\right)}} \approx-\frac{f_{n} I L}{2 k x}=-\frac{f_{n} L}{2 k} A_{I}
$$

Here, it is assumed that $k \gg B A_{I} L$. It can be observed that the fractional frequency change, $\Delta f_{n} / f_{n}=B I L / 2 k x$, is the ratio of the Lorentz force $(B I L)$ over the recovering spring force $(k x)$. Therefore, to maximize the sensitivity of the QFM magnetometer, the Lorentz force needs to be increased whereas the spring force needs to be decreased.

The FM magnetometer has low temperature sensitivity. From (5), the sensitivity $S_{B}$ can be rewritten as:

$$
S_{B}=-\frac{L}{4 \pi \sqrt{k m}} A_{I}
$$

Changes in $Q$ do not directly affect the sensor's frequency output because the sensitivity is independent of $Q$. Only the stiffness $k$ has significant temperature dependence since the resonator's mass $m$ is temperature invariant, the length $L$ varies based on Silicon's thermal coefficient of expansion $(2.6 \mathrm{ppm} / \mathrm{K})$, and the amplifier's gain $A_{I}$ is based on a resistance ratio and is temperature invariant to first-order. Typical temperature dependence of $\sqrt{k}$ for single-crystalline Silicon is $30 \mathrm{ppm} / \mathrm{K}$. The temperature sensitivity of the QFM magnetometer is therefore 10 times smaller than that of a commercially-available AMR compass, which has a temperature sensitivity of $300 \mathrm{ppm} / \mathrm{K}$ compensated by a temperature sensor [8].

\section{LORENTZ FORCE MAGNEMETER}

The magnetometer was fabricated in the epi-seal encapsulation process [9]. The epi-seal encapsulation process was proposed by researchers at the Robert Bosch Research and Technology Center in Palo Alto and then demonstrated in a close collaboration with Stanford University. This collaboration is continuing to develop improvements and extensions to this process for many applications, while the baseline process has been brought into commercial production by SiTime Inc.

Figure 2 shows the Lorentz force magnetometer, a three-port MEMS resonator with electrodes DR for electrostatic driving and electrodes $\mathrm{S}$ for capacitive sensing. The $370 \mu \mathrm{m}$ by $230 \mu \mathrm{m}$ resonator is made out of $40-\mu \mathrm{m}$ thick $<100>$ single crystalline silicon. The suspended MEMS resonator is anchored at the $I B$ electrodes. Bias current $I$ is applied between the $I B+$ and $I B$ electrodes, out of phase with the electrostatic force $F_{E}$. Table I shows the device parameters.

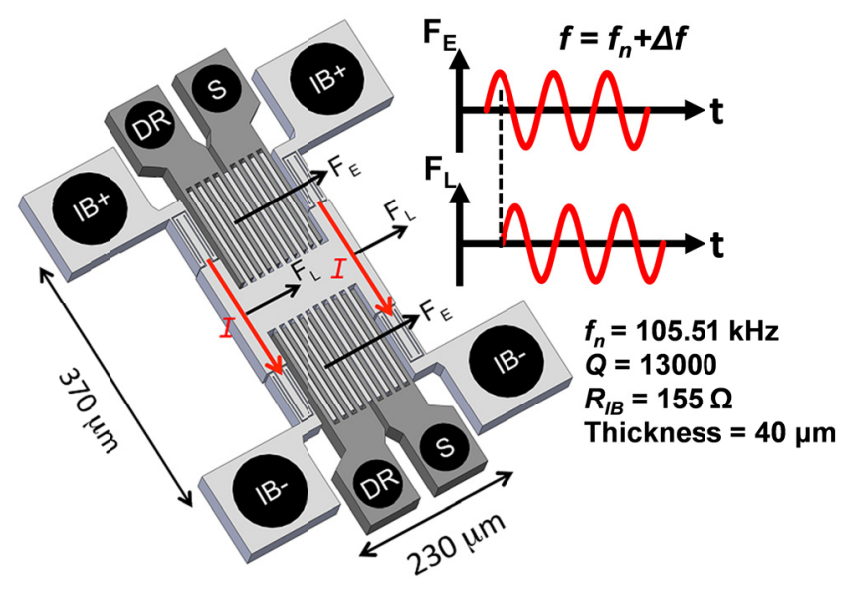

Figure 2: Lorentz force magnetometer.

TABLE I

DEVICE PARAMETERS

\begin{tabular}{ll}
\hline \hline Parameter & Value \\
\hline Device Thickness, t & $40 \mu \mathrm{m}$ \\
Device Area & $0.08 \mathrm{~mm}^{2}$ \\
Proof mass & $4 \mu \mathrm{g}$ \\
Spring Constant, $\mathrm{k}$ & $1800 \mathrm{~N} / \mathrm{m}$ \\
Quality Factor, Q & 13000 \\
Drive Capacitance, $\mathrm{C}_{\mathrm{d}}$ & $660 \mathrm{fF}$ \\
Sense Capacitance, $\mathrm{C}_{\mathrm{s}}$ & $660 \mathrm{fF}$ \\
Capacitance Gap & $0.95 \mu \mathrm{m}$ \\
Resonance Frequency, $f_{n}$ & $105.51 \mathrm{kHz}$ \\
Resistance & $155 \Omega$ \\
Effective Length, $L$ & $250 \mu \mathrm{m}$ \\
\hline \hline
\end{tabular}

\section{IMPLEMENTATION}

The MEMS resonator needs to be first put into a self-sustained oscillation loop, such that the change in the spring constant can be measured by the change in oscillation frequency. Figure 3 shows the block diagram of QFM operation. Here, we use a digital PLL to close the oscillation loop and the phase detector provides an output voltage that is proportional to the change in the oscillation frequency, $\Delta f(B)$. Alternatively, if a hard-limiting amplifier or automatic level control (ALC) are used to close the loop, a frequency counter or one-shot detector can be used to measure

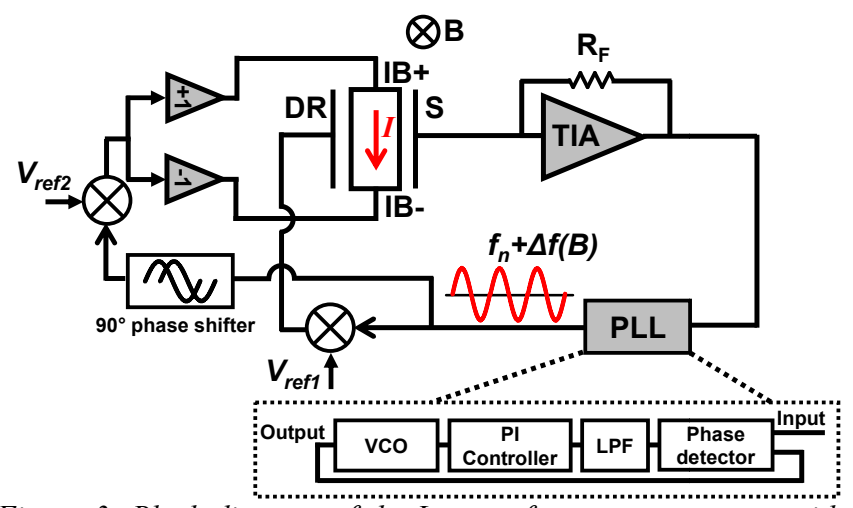

Figure 3: Block diagram of the Lorentz force magnetometer with quadrature FM. Reference voltages $V_{\text {ref } 1}$ and $V_{\text {ref } 2}$ are used to set the amplitude of the electrostatic and Lorentz force components, respectively. 
frequency change.

The bias current driving through the proof-mass to produce Lorentz force $(F=B I L)$ is generated using the electrostatic oscillation signal with a $90^{\circ}$ phase shift. Thus, at resonance, the Lorentz force is always in quadrature with the electrostatic force. The AM and QFM modes can be implemented using the same resonator design with different phase shift $\left(0^{\circ}\right.$ or $\left.90^{\circ}\right)$ between the electrostatic drive and bias current signals.

\section{RESULTS}

Figure 4 shows the output frequency versus input magnetic field of the magnetometer. The magnetometer is driven at a fixed oscillation amplitude of $19 \mathrm{~nm}$. During all measurements, a $0.9 \mathrm{~mA}_{\mathrm{rms}}$ bias current driven through the $155 \Omega$ proof-mass results in $125 \mu \mathrm{W}$ power consumption in the MEMS resonator. The bias current gain is $A_{I}=-6700 \mathrm{~A} / \mathrm{m}$. The measured sensitivity is $500 \mathrm{~Hz} / \mathrm{T}$, close to the theoretical value of $493 \mathrm{~Hz} / \mathrm{T}$. We use customized Helmholtz coils to generate magnetic field for testing. The maximum field is limited to $3.6 \mathrm{mT}$ by the power limit of the coil. The nonlinearity of the sensor is negligible in this range, and the theoretical linear operating range is $2.36 \mathrm{~T}$ (assuming $1 \%$ non-linearity). The inset shows a real-time measurement in response to a $400 \mu \mathrm{T}$ magnetic field input with a frequency of $20 \mathrm{~Hz}$.

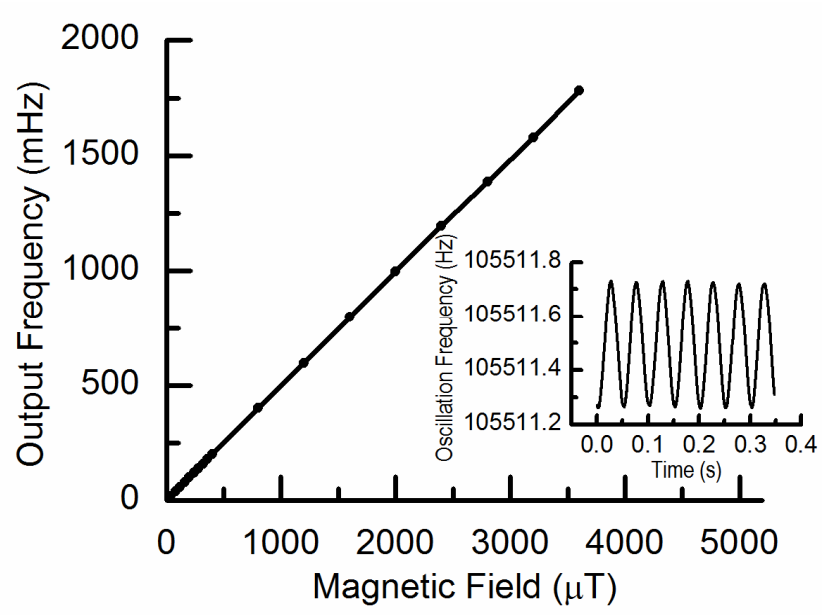

Figure 4. Output frequency vs. input magnetic field of the magnetometer. The measured sensitivity is $500 \mathrm{~Hz} / \mathrm{T}$. Inset: Measured output for $20 \mathrm{~Hz} 400 \mu$ T field input.

Figure 5 shows the frequency response of the magnetometer. Theoretically, the mechanical sensor in FM operation has unlimited bandwidth. Here, to reduce the total noise at the PLL output, the transfer function of the PLL limits the system bandwidth to $50 \mathrm{~Hz}$. The measured sensor response matches well with the predicted transfer function of the digital PLL. The $3 \mathrm{~dB}$ bandwidths for QFM and AM operation are illustrated by the red dotted line. The bandwidth of the magnetometer is extended to $50 \mathrm{~Hz}$ in QFM operation compared to AM operation, in which the bandwidth is only $4 \mathrm{~Hz}$. It has been experimentally shown that the bandwdith is improved by $12 \mathrm{X}$ in the QFM operation compared to the $\mathrm{AM}$ operation, independently from the mechanical sensor bandwidth.

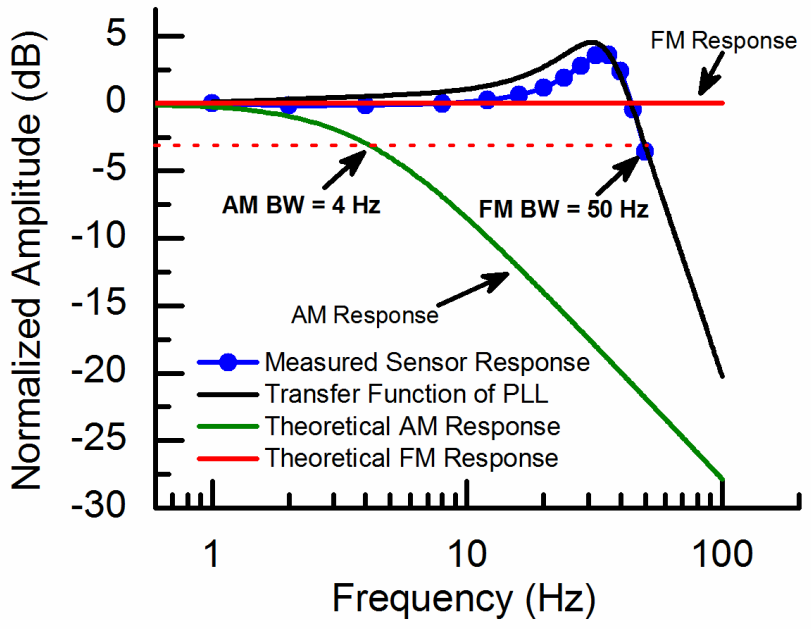

Figure 5. Frequency response of the magnetometer showing QFM achieves $12 x$ greater $B W$ than $A M$ operation. The measured frequency response of the sensor output is shaped by the transfer function of the PLL.

Figure 6 shows the measured oscillator output spectrum in response to input magnetic field $(40 \mu \mathrm{T})$ at $10 \mathrm{~Hz}, 20 \mathrm{~Hz}, 30 \mathrm{~Hz}$ and $40 \mathrm{~Hz}$. The magnetic field appears as double-sided FM sidebands, which is consistent with narrowband frequency modulation. A nearly constant sensitivity over the frequency range is also observed. The slight amplitude difference observed at $30 \mathrm{~Hz}$ and $40 \mathrm{~Hz}$ is due to the frequency response of the PLL which exhibits some gain peaking at these frequencies.

Figure 7 shows the measured noise floor of the magnetometer. Although QFM operation provides constant sensitivity, in a wide sensor bandwidth the noise floor increases as the modulation index decreases. The long-term noise is dominated by the close-to-carrier phase noise of the oscillator, whereas the short-term noise is dominated by the far-from-carrier phase noise. A fitting of the sensor's noise floor shows a $-10 \mathrm{~dB} /$ decade region close-to-carrier, followed by a flat region, and finally a $+20 \mathrm{~dB} /$ decade region far-from-carrier. These three regions correspond to the $1 / f^{3}, 1 / f^{2}$ and white noise components of the phase noise. The loop filter in the

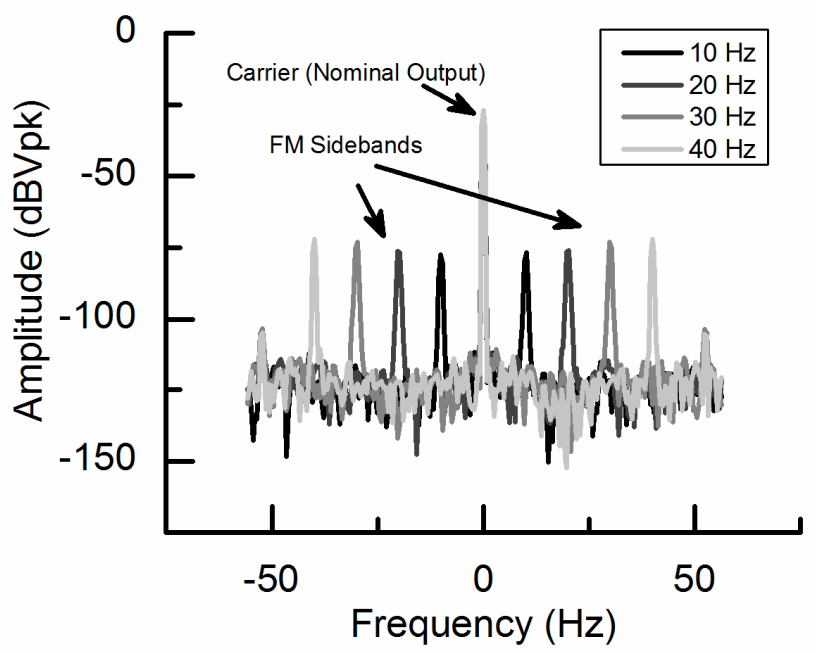

Figure 6. Measured oscillator output spectrum for $10 \mathrm{~Hz}, 20 \mathrm{~Hz}, 30$ $\mathrm{Hz}$ and $40 \mathrm{~Hz}$ magnetic field $(40 \mu \mathrm{T})$. 
PLL will also affect the far-from-carrier phase noise. The noise floor is $500 \mathrm{nT} / \sqrt{\mathrm{Hz}}$ at frequencies from $3 \mathrm{~Hz}$ to $20 \mathrm{~Hz}$, which is comparable to the Brownian-limited noise floor for the same magnetometer operating in AM mode. The dynamic range is expected to be $133 \mathrm{~dB}$. The phase noise is measured as $-92 \mathrm{dBc} / \mathrm{Hz}$ at $10 \mathrm{~Hz}$. When normalized to GSM's $13 \mathrm{MHz}$ [10], the phase noise is $-50 \mathrm{dBc} / \mathrm{Hz}$ at $10 \mathrm{~Hz}$. Table II compares Lorentz force magnetometers with different operation methods.

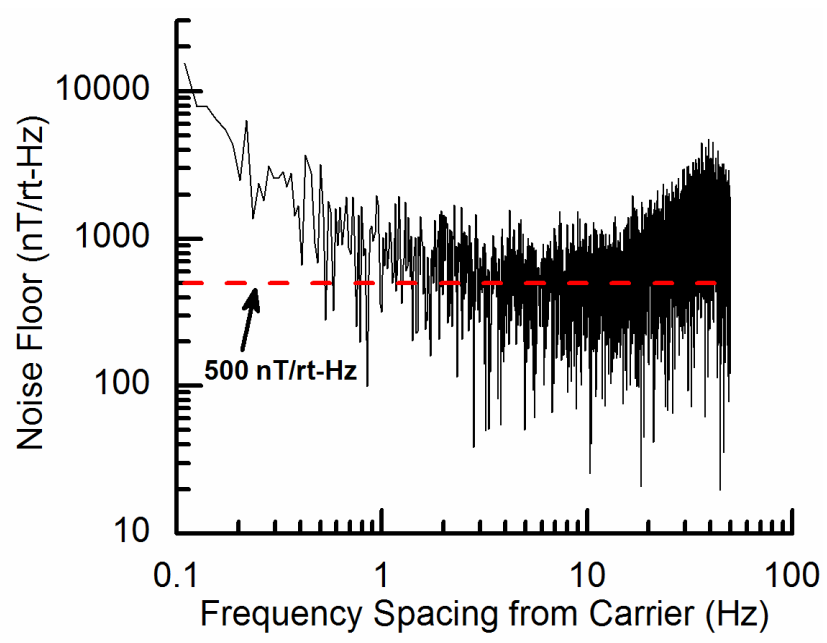

Figure 7. Measured noise floor showing Brownian limited noise from $3 \mathrm{~Hz}$ to $20 \mathrm{~Hz}$.

TABLE II

COMPARISON OF LORENTZ FORCE MAGNETOMETERS

\begin{tabular}{cccc}
\hline \hline \multirow{2}{*}{ Parameter } & \multicolumn{3}{c}{ OPERATION METHODS } \\
\cline { 2 - 4 } & AM & Axial Stress FM & Quadrature FM \\
\hline Readout & Amplitude & Frequency & Frequency \\
Sensitivity & $I L Q / k$ & $A f_{n} I L / k^{*}$ & $f_{n} I L /(2 k x)$ \\
Bandwidth & $f_{n} / 2 Q$ & Unlimited & Unlimited \\
Bias Current & In phase & DC current & Quadrature \\
Brownian- & $\sqrt{4 k_{b} T b}$ & $\sqrt{4 k_{b} T b}$ & $\sqrt{4 k_{b} T b}$ \\
limited & $\frac{I L}{I L}$ & & $\frac{I L}{\text { Resolution }}$ \\
\hline \hline
\end{tabular}

*A is a coupling coefficient that depends on the device geometry.

\section{CONCLUSION}

In conclusion, we demonstrate the QFM operation of a Lorentz force magnetometer based on a $105.5 \mathrm{kHz}$ MEMS oscillator. The magnetometer achieves a sensitivity of $500 \mathrm{~Hz} / \mathrm{T}$ for $0.9 \mathrm{~mA}_{\mathrm{rms}}$ bias current. A Brownian limited noise floor of $500 \mathrm{nT} / \sqrt{\mathrm{Hz}}$ is also measured. Compared to the conventional AM magnetometer, QFM mode provides lower temperature sensitivity, larger sensor bandwidth $(50 \mathrm{~Hz}$ vs. $4 \mathrm{~Hz})$ and larger dynamic range $(2.36 \mathrm{~T}$ vs. $10 \mathrm{mT}$ ), demonstrating great potential for electronic compass applications.

\section{ACKNOWLEDGEMENTS}

This work was supported by the Defense Advanced Research Projects Agency (DARPA) Precision Navigation and Timing program (PNT) managed by Dr. Andrei Shkel and Dr. Robert Lutwak under contract \# N66001-12-1-4260 and the National Science Foundation under award number CMMI-0846379. The work was performed in part at the Stanford Nanofabrication Facility (SNF) which is supported by National Science Foundation through the NNIN under Grant ECS-9731293. The authors would also like to thank the SNF staff, particularly M. M. Stevens for the timely assistance with the epitaxial reactor.

\section{REFERENCES}

[1] M. Li, E. J. Ng, V. A. Hong, C. H. Ahn, Y. Yang, T. W. Kenny, and D. A. Horsley, "Lorentz force magnetometer using a micromechanical oscillator," Applied Physics Letters, vol. 103(173504), 2013.

[2] W. Zhang and J. E. Y. Lee, "Characterization and model validation of a micromechanical resonant magnetic field sensor," in Proc. Transducers, 2013, pp. 1859-1862.

[3] M. Li, E. J. Ng, V. A. Hong, C. H. Ahn, Y. Yang, T. W. Kenny, and D. A. Horsley, "Single-structure 3-axis Lorentz force magnetometer with sub-30 nT/ $\sqrt{\mathrm{HZ}}$ resolution," in Proc. MEMS 2014, pp. 80-83.

[4] AK8975 3-Axis Electronic Compass, Asahi Kasei Microdevices Semiconductor Inc, Tokyo, Japan. 2010.

[5] W. Zhang and J. E. Y. Lee, "Frequency-based magnetic field sensing using Lorentz force axial strain modulation in a double-ended tuning fork," Sensors and Actuators A: Physical, to be published.

[6] M. H. Kline, Y. Yeh, B. Eminoglu, H. Najar, M. Daneman, D. A. Horsley, and B. E. Boser, "Quadrature FM gyroscope," in Proc. MEMS 2013, pp. 604-608.

[7] R. Sunier, T. Vancura, Y. Li, K. U. Kirstein, H. Baltes, and O. Brand, "Resonant Magnetic Field Sensor With Frequency Output," Journal of Microelectromechanical Systems, vol. 15, pp. 1098-1107, 2006.

[8] 3-Axis Digital Compass IC HMC5983 Honeywell Inc., Plymouth, MN, 2012.

[9] R. N. Candler, M. A. Hopcroft, B. Kim, W.-T. Park, R. Melamud, M. Agarwal, G. Yama, A. Partridge, M. Lutz, and T. W. Kenny, "Long-Term and Accelerated Life Testing of a Novel Single-Wafer Vacuum Encapsulation for MEMS Resonators," Journal of Microelectromechanical Systems, vol. 15, pp. 1446-1456, 2006.

[10] T. L. Naing, T. O. Rocheleau, E. Alon, and C. T. C. Nguyen, "A 78-microwatt GSM phase noise-compliant pierce oscillator referenced to a 61-MHz wine-glass disk resonator," in European Frequency and Time Forum \& International Frequency Control Symposium (EFTF/IFC), 2013, pp. 562-565.

\section{CONTACT}

*Mo Li, tel: +1-530-752-5180; moxli@ucdavis.edu 